\title{
Optimized Buffer Stack with Carbon-Doping for Performance Improvement of GaN HEMTs
}

\author{
Ajay Shanbhag ${ }^{1}$, Sruthi M P ${ }^{1}$, Farid Medjdoub ${ }^{2}$, Anjan Chakravorty ${ }^{1}$, Nandita DasGupta ${ }^{1}$, and \\ Amitava DasGupta ${ }^{1}$ \\ ${ }^{1}$ Department of Electrical Engineering, Indian Institute of Technology Madras, India \\ ${ }^{2}$ IEMN-CNRS, Institute of Electronics, Microelectronics and Nanotechnology, France
}

\begin{abstract}
This paper focuses on determining an optimized value of carbon-doping level in the buffer and corresponding channel thickness to improve the performance of GaN HEMTs in terms of subthreshold slope $(S S)$, breakdown voltage $\left(V_{B D}\right)$ and transit frequency $\left(f_{t}\right)$. With the increase in carbon-doping, we observe improvements in $S S$ and $V_{B D}$ while the $f_{t}$ is reduced. However, as the channel thickness increases above a certain thickness, no significant impact of carbon-doping is observed on the characteristics. TCAD simulation is calibrated using experimental data for a device with carbon-doping level of $3 \times 10^{18} \mathrm{~cm}^{-3}$ in the buffer with channel thickness of $\mathbf{5 0 0} \mathbf{~ n m}$. Using the calibrated device in TCAD, the carbon-doping level and channel thickness are varied to see the effects on different parameters. We observed that an optimized channel thickness of $200 \mathrm{~nm}$ with carbon-doping level of $1 \times 10^{19} \mathrm{~cm}^{-3}$ in the buffer yields the best results in terms of $V_{B D}$ and $f_{t}$.
\end{abstract}

Index Terms-AIGaN-GaN HEMTs, AlInN-GaN HEMTs, breakdown voltage, buffer traps, C-doping, subthreshold slope, transit frequency.

\section{INTRODUCTION}

$\mathbf{W}$ ITH the increased demand for high power and RF applications, Gallium Nitride (GaN) high electron mobility transistors (HEMTs) have proven to be an ideal choice due to its material properties such as large band gap, high electron mobility, saturation velocity, and electric field strength, along with superior thermal conductivity [1]-[4]. As the unintentional dopants incorporated during the epitaxial growth of $\mathrm{GaN}$, the buffer leakage increases, causing a deterioration of the device breakdown voltage $\left(V_{B D}\right)$, subthreshold slope $(S S)$, and on-off current ratio $\left(I_{O N} / I_{O F F}\right)$. Various techniques have been reported to reduce the buffer leakage, such as intentional doping with acceptor traps such as Iron $(\mathrm{Fe})$ or Carbon $(\mathrm{C})$ [5], and growing a high bandgap material such as AlGaN below the channel layer to ensure a backbarrier [6]. We initially focus on reducing the buffer leakage and increase buffer resistivity by intentionally doping carbon in the buffer layer. Although this helps in increasing $\left(V_{B D}\right)$, the introduction of such traps degrades the dynamic performance of the device due to the slow emission process of the traps, thereby reducing the transit frequency $\left(f_{t}\right)$. Hence, an

The authors would like to thank Centre for NEMS and Nanophotonics (CNNP), IIT Madras and Science and Engineering Research Board (SERB), Govt. of India vide project no.CRG/2018/001081 for partial financial support and Veeco Instruments Inc. for providing the $\mathrm{GaN}$ wafers for experimental purposes. optimized buffer stack is necessary to get the best performance from the device.

Few works have reported the use of C-doping in $\mathrm{GaN}$ as a deep acceptor [7], [8]. In contrast, a few others have reported it to be self-compensating in nature since $\mathrm{C}$ acts as a deep acceptor and a shallow donor as it can replace $\mathrm{Ga}$ or $\mathrm{N}$ and acts as either donor or acceptor, respectively [9], [10]. However, if we consider an equal concentration of acceptor and donor, no current collapse should be observed unlike experimental data. Additionally, the energy required for $\mathrm{C}$ to replace $\mathrm{Ga}$ in $\mathrm{GaN}$ is high, resulting in a higher probability of it behaving like an acceptor trap [8]. Hence in the TCAD simulations, we have considered $80 \%$ self-compensating behavior.

As carbon acts as deep acceptor trap centers in $\mathrm{GaN}$, it can potentially trap the electrons from 2-dimensional electron gas $(2 D E G)$ in the buffer, degrading the DC characteristics significantly. A sudden change in the drain and /or gate voltage in pulsed applications generates a sudden change in the electric field at the gate edge on the drain side, resulting in enough energy for the electrons from the channel to go deeper into the buffer and get trapped in the trap sites. As these are deep traps, the emission process is slow, resulting in trapping-related dispersion effects such as drain-lag, gate-lag, and current collapse [11], [12]. Hence, it is a common practice to dope carbon away from the channel region to prevent the trapping of electrons from the $2 D E G$. However, if the $\mathrm{C}$-doped layer is far away from the channel region, the buffer leakage increases. Hence, the channel region must not be very thick. Also, the carbon concentration in the C-doped layer is an important parameter to control the leakage current. If the carbon concentration is very high, the dynamic performance of the device will degrade due to its slow detrapping process. Hence, an optimum carbon concentration and channel thickness are required to obtain the best results.

Although studies on the impact of $V_{B D}$ with C-doping are available in the literature [13], a detailed and systematic research on the interplay of the channel thickness and Cdoping on the device performance is missing. In this paper, we focus on the variation of channel thickness and C-doping level and compare its impact on $S S, I_{O N} / I_{O F F}, V_{B D}$, and $f_{t}$. Section II discusses the TCAD simulation framework including the detailed analysis of channel thickness and Cdoping level on the device performance. Obtained results are analyzed in detail with appropriate physical insights. Finally, we conclude in Section III. 


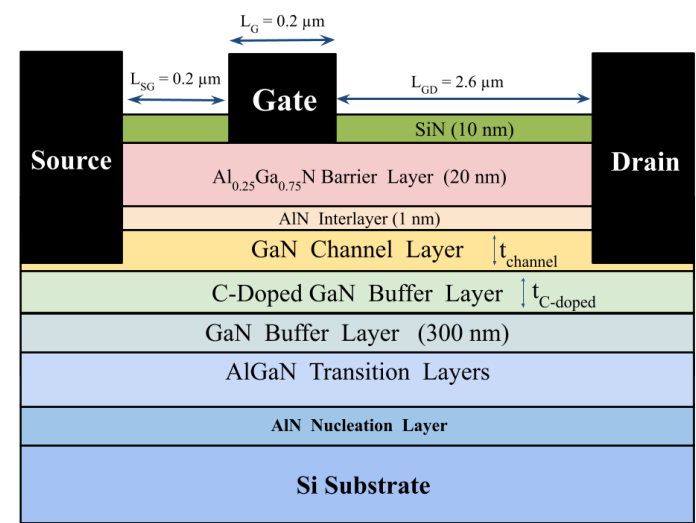

Fig. 1: Cross-sectional view of the device under investigation.

\section{TCAD SimULATION FrAMEWORK AND RESUlTS}

\section{A. Device structure and models}

The GaN HEMT structure as shown in Fig. 1 is calibrated in TCAD using Synopsys Sentaurus [14]. The device consists of $\mathrm{Al}_{0.25} \mathrm{Ga}_{0.75} \mathrm{~N} / \mathrm{GaN}$ heterostructure on a silicon ( $\mathrm{Si}$ ) substrate. The epilayer structure includes $20 \mathrm{~nm}$ AlGaN barrier layer, 1 $\mathrm{nm}$ AlN spacer, $500 \mathrm{~nm}$ thick GaN channel $\left(t_{\text {channel }}\right), 200$ $\mathrm{nm}$ C-doped layer $\left(t_{C-\text { doped }}\right)$ with concentration of $3 \times 10^{18}$ $\mathrm{cm}^{-3}$, and $300 \mathrm{~nm} \mathrm{GaN}$ buffer layer followed by AlGaN transition layer and $\mathrm{Si}$ substrate. The HEMT is fabricated with gate length $\left(L_{G}\right)$ of $0.2 \mu \mathrm{m}$, source-gate spacing $\left(L_{S G}\right)$ of $0.2 \mu \mathrm{m}$, gate-drain spacing $\left(L_{G D}\right)$ of $2.6 \mu \mathrm{m}$, and 50 $\mu \mathrm{m}$ gate width. To account for the surface states, surface donor traps are considered at an energy level of $E_{C}-0.5$ $\mathrm{eV}$ with a density of $3 \times 10^{13} \mathrm{~cm}^{-2}$ as reported in [15]. Using deep level transient spectroscopy (DLTS) and low-frequency $\mathrm{Y}_{22}$ parameters, it has been shown that the C-traps have an activation energy of around $0.9 \mathrm{eV}$ from the valence band [16] and the same has been used in simulation with a concentration of $3 \times 10^{18} \mathrm{~cm}^{-3}$, and electron and hole capture cross-section as $10^{-18} \mathrm{~cm}^{2}$ and $10^{-14} \mathrm{~cm}^{2}$ respectively in the C-doped layer. To account for the self compensating nature, shallow donors with a concentration of $2.4 \times 10^{18} \mathrm{~cm}^{-3}$ with activation energy of $0.2 \mathrm{eV}$ from conduction band with similar electron and hole capture cross-section have been considered. In other regions of the device, acceptor traps with a concentration of $5 \times 10^{16} \mathrm{~cm}^{-3}$ and activation energy of $0.47 \mathrm{eV}$ from the conduction band are considered. The unintentional n-doping in $\mathrm{GaN}$ is considered to be in the order of $10^{15} \mathrm{~cm}^{-3}$. The work function of the gate Schottky contact is set to 4.4 $\mathrm{eV}$. To make the source and drain contacts ohmic, non-local electron tunneling at the contacts is activated by setting a work function of $4 \mathrm{eV}$. The polarization model is used to consider the polarization charges at the interfaces. The drift-diffusion equations are solved along with various physical models, e.g., doping and field-dependent mobility, mobility degradation due to traps, thermionic emission, Shockley-Read-Hall (SRH) recombination, and Fermi statistics. According to Chynoweth law, the impact ionization model has been considered in $\mathrm{GaN}$ to account for the carrier generation. The breakdown voltage
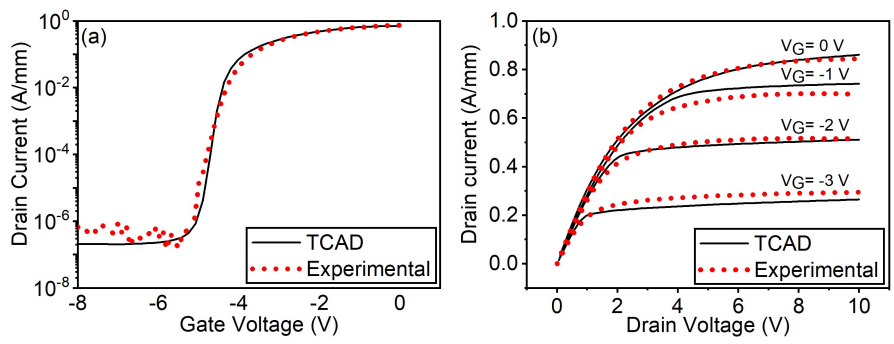

Fig. 2: Comparison of results obtained from experimental data and TCAD simulations: (a) transfer and (b) output characteristics of GaN HEMT.

TABLE I: Reference Device Metric Values

\begin{tabular}{|l|l|}
\hline Device Metric & Value \\
\hline Maximum drain current $\left(I_{D \max }\right)$ & $45.38 \mathrm{~mA}$ \\
On-off current ratio $\left(I_{O N} / I_{O F F}\right)$ & $4.6 \times 10^{6}$ \\
Subthreshold slope $(S S)$ & $128 \mathrm{mV} / \mathrm{dec}$ \\
Breakdown voltage $\left(V_{B D}\right)$ & $119 \mathrm{~V}$ \\
Transit frequency $\left(f_{t}\right)$ & $43.8 \mathrm{Ghz}$ \\
\hline
\end{tabular}

is measured as the OFF-state drain current of $1 \mathrm{~mA} / \mathrm{mm}$ with gate-source voltage $\left(V_{G S}\right)$ being set at $-8 \mathrm{~V}$.

Using the models and parameter values mentioned so far, detailed TCAD simulation is carried out. Corresponding simulation results along with the experimental data for the transfer and output characteristics are shown in Fig. 2. We observe that the on-state current and leakage current have been wellcalibrated. For this reference device, the observed values of $S S, I_{O N} / I_{O F F}, V_{B D}$, and $f_{t}$ are shown in Table I. In the following subsections, we attempt to optimize C-doping level in the GaN buffer layer and $t_{\text {channel }}$ by comparing the resulting metrics with those of the reference device.

\section{B. Channel thickness variation with fixed C-doping level}

From the reference parameter values of the device, we varied $t_{\text {channel }}$ keeping C-doping level fixed at $3 \times 10^{18}$ $\mathrm{cm}^{-3}$. Corresponding variations of device metric are presented in Fig. 3 and Table II. On reducing the channel thickness from $500 \mathrm{~nm}$ to $35 \mathrm{~nm}$, the ratio $I_{O N} / I_{O F F}$ increases by six orders, $S S$ reduces by $45 \mathrm{mV} / \mathrm{dec}$, and $V_{B D}$ increases more than four times, while the $f_{t}$ is reduced by half as compared to those of the reference device. As the channel region thickness is reduced, the $\mathrm{C}-\mathrm{GaN}$ layer comes closer
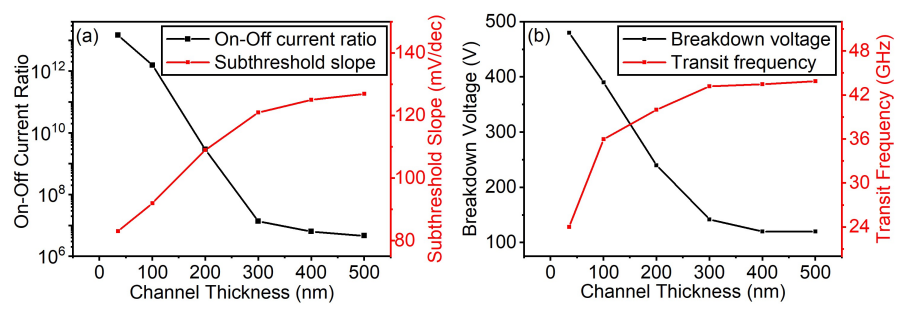

Fig. 3: TCAD results showing variation of channel thickness on: (a) On-off current ratio and subthreshold slope, (b) breakdown voltage and transit frequency, by keeping C-doping at 3 $\times 10^{18} \mathrm{~cm}^{-3}$ in C-doped GaN buffer. 
TABLE II: Device Metric Values with C-doping in C-GaN as $3 \times 10^{18} \mathrm{~cm}^{-3}$

\begin{tabular}{|l|l|l|l|l|l|}
\hline $\begin{array}{l}t_{\text {channel }} \\
(\mathrm{nm})\end{array}$ & $\begin{array}{l}I_{\text {Dmax }} \\
(\mathrm{mA})\end{array}$ & $I_{O N} / I_{\text {OFF }}$ & $\begin{array}{l}S S \\
(\mathrm{mV} / \mathrm{dec})\end{array}$ & $\begin{array}{l}V_{B D} \\
(\mathrm{~V})\end{array}$ & $\begin{array}{l}f_{t} \\
(\mathrm{GHz})\end{array}$ \\
\hline 35 & 36 & $1.45 \times 10^{13}$ & 83 & 480 & 24.5 \\
100 & 42.5 & $1.57 \times 10^{12}$ & 92 & 389 & 36 \\
200 & 44.9 & $2.9 \times 10^{9}$ & 109 & 239 & 40 \\
300 & 45.2 & $1.38 \times 10^{7}$ & 121 & 142 & 43.2 \\
400 & 45.3 & $6.35 \times 10^{6}$ & 125.57 & 119 & 43.5 \\
500 & 45.4 & $4.65 \times 10^{6}$ & 127 & 119 & 43.8 \\
\hline
\end{tabular}

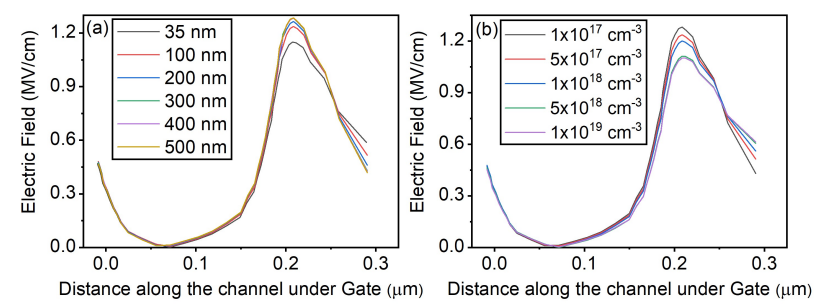

Fig. 4: TCAD results showing the effect on electric field profile below the gate from drain to source by varying (a) channel thickness with C-Doping level of $3 \times 10^{18} \mathrm{~cm}^{-3}$ in $\mathrm{C}-\mathrm{GaN}$ and (b) C-doping level with channel thickness of $35 \mathrm{~nm}$.

to the channel, causing a significant reduction of the offstate leakage. This results in higher $I_{O N} / I_{O F F}$ and better $S S$. Additionally, we observe electric field relaxation at the drain side of the gate edge as shown in Fig. 4a due to which $V_{B D}$ increases. However, the trapping increases significantly, reducing the overall $f_{t}$. From the simulated data, we see that as the channel thickness increases above $300 \mathrm{~nm}$, there is no significant change in device metrics compared to those of the reference device. This is due to a reduced interaction of C-dopants with $2 D E G$ leading to an insignificant effect on the electric field as observed in Fig. 4a. However, with a reduction in channel thickness, we see a few metrics are significantly improved while a few others are deteriorated. Hence, we attempt to optimize the C-doping level in the C-doped GaN buffer layer in order to obtain an optimum performance concerning all the metrics.

\section{C-doping level variation for different channel thickness}

To find an optimum buffer design with C-doped $\mathrm{GaN}$, we varied the $\mathrm{C}$-doping level in the $\mathrm{C}$-doped $\mathrm{GaN}$ buffer for various channel thicknesses. Fig. 5 shows the effects of Cdoping levels for various channel thicknesses on different metrics.

Table III shows the results of different metrics for $t_{\text {channel }}$ of $35 \mathrm{~nm}$. It is observed that if the C-doping level is below

TABLE III: Device Metric Values with $t_{\text {channel }}$ as $35 \mathrm{~nm}$

\begin{tabular}{|l|l|l|l|l|l|}
\hline $\begin{array}{l}\text { C-Doping in C- } \\
\text { GaN }\left(\mathrm{cm}^{-3}\right)\end{array}$ & $\begin{array}{l}I_{D \max } \\
(\mathrm{mA})\end{array}$ & $I_{\text {ON }} / I_{\text {OFF }}$ & $\begin{array}{l}S S \\
(\mathrm{mV} / \mathrm{dec})\end{array}$ & $\begin{array}{l}V_{B D} \\
(\mathrm{~V})\end{array}$ & $\begin{array}{l}f_{t} \\
(\mathrm{GHz})\end{array}$ \\
\hline $1 \times 10^{17}$ & 45.4 & $3.8 \times 10^{4}$ & 279 & 55 & 44.3 \\
$5 \times 10^{17}$ & 42.5 & $1.1 \times 10^{12}$ & 105 & 205 & 38 \\
$1 \times 10^{18}$ & 39 & $1.1 \times 10^{12}$ & 84.6 & 317 & 33.7 \\
$5 \times 10^{18}$ & 34 & $1.1 \times 10^{12}$ & 80.4 & 550 & 12.9 \\
$1 \times 10^{19}$ & 30 & $1.1 \times 10^{12}$ & 79 & 670 & 12 \\
\hline
\end{tabular}

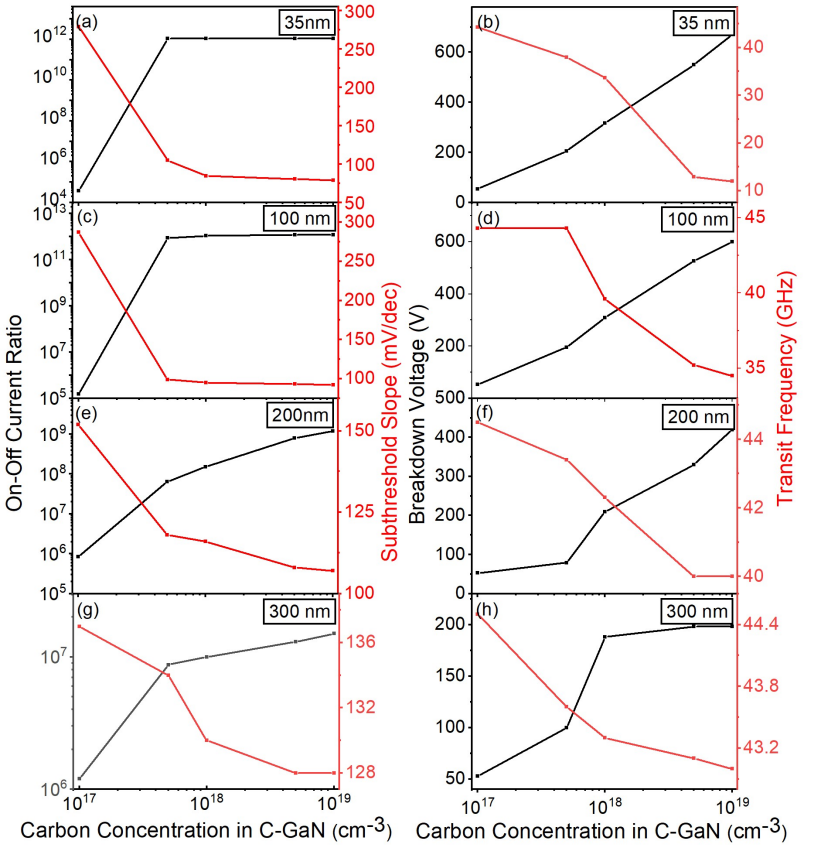

Fig. 5: TCAD results showing the effects of variation of $\mathrm{C}$ doping in C-GaN buffer for different channel thickness on different device metrics such as on-off current ratio, subthreshold slope, breakdown voltage, and transit frequency.

$5 \times 10^{17} \mathrm{~cm}^{-3}, S S, I_{O N} / I_{O F F}$, and $V_{B D}$ deteriorate significantly, due to the self-compensating nature of carbon in GaN. As a result, we see an overall reduced concentration of acceptor traps causing an increase in the leakage current. However, as the C-concentration is increased above $5 \times 10^{17}$ $\mathrm{cm}^{-3}$, we see improvements in $S S$ and $V_{B D}$, but with a significant fall in $f_{t}$. Due to a reduction in the leakage current as the C-doping level increases and effective confinement of $2 D E G$ in the channel region, we find improvements in $S S$ and $I_{O N} / I_{O F F}$. Additionally due to the electric field relaxation at the drain side of gate edge as shown in Fig. $4 \mathrm{~b}, V_{B D}$ improves significantly. But as $\mathrm{C}-\mathrm{GaN}$ layer is very close to the channel, trapping effect is higher, causing the $f_{t}$ to fall. Hence, considering $t_{\text {channel }}$ as $35 \mathrm{~nm}$, it is suggested that C-doping level should be around $5 \times 10^{17} \mathrm{~cm}^{-3}$ in order to ensure the best performance. However, the value of $f_{t}$ is fairly low and the improvement in $V_{B D}$ is only by a factor of 2 with this configuration. Hence, it is decided to increase $t_{\text {channel }}$ to $100 \mathrm{~nm}$. Table IV shows the resulting metric values.

We observe a similar trend at a lower C-doping level. However, as the doping level increases, the \% reduction in $f_{t}$ is less compared to the previous case, as the electrons from

TABLE IV: Device Metric Values with $t_{\text {channel }}$ as $100 \mathrm{~nm}$

\begin{tabular}{|l|l|l|l|l|l|}
\hline $\begin{array}{l}\text { C-Doping in C- } \\
\text { GaN }\left(\mathrm{cm}^{-3}\right)\end{array}$ & $\begin{array}{l}I_{D \max } \\
(\mathrm{mA})\end{array}$ & $I_{O N} / I_{O F F}$ & $\begin{array}{l}S S \\
(\mathrm{mV} / \mathrm{dec})\end{array}$ & $\begin{array}{l}V_{B D} \\
(\mathrm{~V})\end{array}$ & $\begin{array}{l}f_{t} \\
(\mathrm{GHz})\end{array}$ \\
\hline $1 \times 10^{17}$ & 45.5 & $1.5 \times 10^{5}$ & 287 & 53 & 44.3 \\
$5 \times 10^{17}$ & 44 & $8.8 \times 10^{11}$ & 98.9 & 196 & 44.3 \\
$1 \times 10^{18}$ & 43.4 & $1.2 \times 10^{12}$ & 95.5 & 309 & 39.7 \\
$5 \times 10^{18}$ & 42.2 & $1.2 \times 10^{12}$ & 93.2 & 527 & 35.2 \\
$1 \times 10^{19}$ & 42 & $1.2 \times 10^{12}$ & 92 & 600 & 34.4 \\
\hline
\end{tabular}


TABLE V: Device Metric Values with $t_{\text {channel }}$ as $200 \mathrm{~nm}$

\begin{tabular}{|l|l|l|l|l|l|}
\hline $\begin{array}{l}\text { C-Doping in C- } \\
\mathrm{GaN}\left(\mathrm{cm}^{-3}\right)\end{array}$ & $\begin{array}{l}I_{D \max }(\mathrm{mA}) \\
I_{O N} / I_{\text {OFF }}\end{array}$ & $\begin{array}{l}S S \\
(\mathrm{mV} / \mathrm{dec})\end{array}$ & $\begin{array}{l}V_{B D} \\
(\mathrm{~V})\end{array}$ & $\begin{array}{l}f_{t} \\
(\mathrm{GHz})\end{array}$ \\
\hline $1 \times 10^{17}$ & 45.5 & $8.54 \times 10^{5}$ & 152 & 53 & 44.5 \\
$5 \times 10^{17}$ & 45 & $6.4 \times 10^{7}$ & 118 & 78.9 & 43.34 \\
$1 \times 10^{18}$ & 44.9 & $1.5 \times 10^{8}$ & 116 & 209 & 42.3 \\
$5 \times 10^{18}$ & 44.78 & $8.1 \times 10^{8}$ & 116 & 330 & 40 \\
$1 \times 10^{19}$ & 44.8 & $1.2 \times 10^{9}$ & 107 & 440 & 40 \\
\hline
\end{tabular}

TABLE VI: Device Metric Values with $t_{\text {channel }}$ as $300 \mathrm{~nm}$

\begin{tabular}{|l|l|l|l|l|l|}
\hline $\begin{array}{l}\text { C-Doping in C- } \\
\text { GaN }\left(\mathrm{cm}^{-3}\right)\end{array}$ & $\begin{array}{l}I_{D \max } \\
(\mathrm{mA})\end{array}$ & $I_{O N} / I_{O F F}$ & $\begin{array}{l}S S \\
(\mathrm{mV} / \mathrm{dec})\end{array}$ & $\begin{array}{l}V_{B D} \\
(\mathrm{~V})\end{array}$ & $\begin{array}{l}f_{t} \\
(\mathrm{GHz})\end{array}$ \\
\hline $1 \times 10^{17}$ & 45.5 & $1.2 \times 10^{6}$ & 137 & 53 & 44.5 \\
$5 \times 10^{17}$ & 45.3 & $8.7 \times 10^{6}$ & 134 & 100 & 43.6 \\
$1 \times 10^{18}$ & 45.3 & $1 \times 10^{7}$ & 130 & 188 & 43.3 \\
$5 \times 10^{18}$ & 45.3 & $1.3 \times 10^{7}$ & 128 & 198 & 43.1 \\
$1 \times 10^{19}$ & 45.3 & $1.5 \times 10^{7}$ & 128 & 198 & 43 \\
\hline
\end{tabular}

the $2 D E G$ are no longer very close to the $\mathrm{C}-\mathrm{GaN}$ layer. From the simulation results, we suggest an optimum buffer design considering $t_{\text {channel }}$ as $100 \mathrm{~nm}$ and C-doping level of the order of $1 \times 10^{18} \mathrm{~cm}^{-3}$ in $\mathrm{C}-\mathrm{GaN}$ layer to achieve the best results in terms of all the metrics. Though $V_{B D}$ increases with higher $\mathrm{C}$ doping, it is not preferred for pulsed applications, as it would lead to high current collapse. For this optimized device, $V_{B D}$ is around 2.5 times higher than the reference device, while $f_{t}$ is only marginally reduced. Next we simulated devices with $t_{\text {channel }}$ of $200 \mathrm{~nm}$ to achieve a higher $V_{B D}$ value without compensating for $f_{t}$. The corresponding results for the various metrics are shown in Table $\mathrm{V}$.

In this case, as C-doping level is increased, an even lesser impact on $f_{t}$ is observed. On the other hand, there is a fourfold increase in $V_{B D}$ over that of the reference device. Also, the leakage current has decreased by 3 orders of magnitude. Considering $t_{\text {channel }}$ as $200 \mathrm{~nm}$ and C-doping of around $1 \times$ $10^{19} \mathrm{~cm}^{-3}$ in $\mathrm{C}-\mathrm{GaN}$ buffer, we obtain the best performance in all the metrics. Simulation was also carried out by considering $t_{\text {channel }}$ fixed at $300 \mathrm{~nm}$ and varying the C-doping level to improve the metrics. Table VI shows the corresponding results where we find hardly any change in $f_{t}$ with increase in Cdoping. However, $V_{B D}$ has increased to only around 1.6 times that of the reference device for a $\mathrm{C}$-doping concentration of $5 \times 10^{18} \mathrm{~cm}^{-3}$ or higher. The simulation results for $t_{\text {channel }}$ of $400 \mathrm{~nm}$ and $500 \mathrm{~nm}$ with varied C-doping levels in C-GaN do not show any significant improvement over the reference device metrics. Hence they are not shown here.

\section{CONCLUSION}

We have studied the interplay between GaN channel thickness and carbon-doping in the GaN buffer layer using calibrated TCAD simulations. It is observed that as the channel thickness decreases keeping the C-doping unaltered, $S S$, $I_{O N} / I_{O F F}$, and $V_{B D}$ improve while $f_{t}$ reduces significantly. We observe a similar trend as the C-doping level is increased for a given channel thickness. Additionally, as the channel layer thickness increases beyond $300 \mathrm{~nm}$, no significant impact on the above parameters is observed. Analyzing the results obtained from simulations, we obtain an optimized structure with $200 \mathrm{~nm}$ GaN channel thickness followed by a C-GaN layer with a C-doping of $1 \times 10^{19} \mathrm{~cm}^{-3}$. The resulting device yields the best values for $S S, I_{O N} / I_{O F F}, V_{B D}$, and $f_{t}$. Our study and corresponding findings can help the industries and academia to obtain an optimum set of buffer layer parameters during its epitaxial growth to extract the best results for both high power and RF applications.

\section{REFERENCES}

[1] M. Asif Khan, A. Bhattarai, J. Kuznia, and D. Olson, "High electron mobility transistor based on a GaN-AlGaN heterojunction," Applied Physics Letters, vol. 63, no. 9, pp. 1214-1215, 1993. DOI : 10.1063/1.109775.

[2] T. Palacios, A. Chakraborty, S. Rajan, C. Poblenz, S. Keller, S. DenBaars, J. Speck, and U. Mishra, "High-power AlGaN/GaN HEMTs for ka-band applications," IEEE Electron Device Letters, vol. 26, no. 11, pp. 781-783, 2005. DOI : 10.1109/LED.2005.857701.

[3] T. Zhang, J. Zhang, H. Zhou, Y. Zhang, T. Chen, K. Zhang, Y. Wang, K. Dang, Z. Bian, X. Duan, et al., "High-performance lateral GaN Schottky barrier diode on silicon substrate with low turn-on voltage of $0.31 \mathrm{~V}$, high breakdown voltage of $2.65 \mathrm{kV}$ and high-power figureof-merit of 2.65 GW cm- 2," Applied Physics Express, vol. 12, no. 4, p. 046502, 2019. DOI : 10.7567/1882-0786/ab0712.

[4] N. Remesh, N. Mohan, S. Raghavan, R. Muralidharan, and D. N. Nath, "Optimum Carbon Concentration in GaN-on-Silicon for Breakdown Enhancement in AlGaN/GaN HEMTs," IEEE Transactions on Electron Devices, vol. 67, no. 6, pp. 2311-2317, 2020. DOI : 10.1109/TED.2020. 2989421.

[5] S. Kato, Y. Satoh, H. Sasaki, I. Masayuki, and S. Yoshida, "Cdoped $\mathrm{GaN}$ buffer layers with high breakdown voltages for highpower operation AlGaN/GaN HFETs on 4-in Si substrates by MOVPE," Journal of Crystal Growth, vol. 298, pp. 831-834, 2007. DOI : 10.1016/j.jcrysgro.2006.10.192.

[6] N. Onojima, N. Hirose, T. Mimura, and T. Matsui, "High off-state breakdown voltage 60 -nm-long-gate $\mathrm{AlGaN} / \mathrm{GaN}$ heterostructure fieldeffect transistors with AlGaN back-barrier," Japanese Journal of Applied Physics, vol. 48, no. 9R, p. 094502, 2009. DOI : 10.1143/JJAP.48. 094502 .

[7] J. Lyons, A. Janotti, and C. Van de Walle, "Carbon impurities and the yellow luminescence in GaN," Applied Physics Letters, vol. 97, no. 15, p. 152108, 2010. DOI : 10.1063/1.3492841.

[8] J. Lyons, A. Janotti, and C. Van de Walle, "Effects of carbon on the electrical and optical properties of InN, GaN, and AlN," Physical Review $B$, vol. 89, no. 3, p. 035204, 2014. DOI : 10.1103/PhysRevB.89.035204.

[9] A. Fariza, A. Lesnik, J. Bläsing, M. P. Hoffmann, F. Hörich, P. Veit, H. Witte, A. Dadgar, and A. Strittmatter, "On reduction of current leakage in GaN by carbon-doping," Applied Physics Letters, vol. 109, no. 21 , p. 212102 , 2016. DOI : $10.1063 / 1.4968823$.

[10] B. Rackauskas, M. J. Uren, S. Stoffels, M. Zhao, S. Decoutere, and M. Kuball, "Determination of the self-compensation ratio of carbon in AlGaN for HEMTs," IEEE Transactions on Electron Devices, vol. 65, no. 5, pp. 1838-1842, 2018. DOI : 10.1109/TED.2018.2813542.

[11] C. Miccoli, V. C. Martino, S. Reina, and S. Rinaudo, "Trapping and thermal effects analysis for AlGaN/GaN HEMTs by means of TCAD simulations," IEEE Electron Device Letters, vol. 34, no. 9, pp. 11211123, 2013. DOI : 10.1109/LED.2013.2274326.

[12] W. Hu, X. Chen, F. Yin, J. Zhang, and W. Lu, "Two-dimensional transient simulations of drain lag and current collapse in GaN-based high-electron-mobility transistors," Journal of Applied Physics, vol. 105, no. 8, p. 084502, 2009. DOI : 10.1063/1.3106603.

[13] M. J. Uren, M. Caesar, S. Karboyan, P. Moens, P. Vanmeerbeek, and M. Kuball, "Electric field reduction in C-doped AlGaN/GaN on Si high electron mobility transistors," IEEE Electron Device Letters, vol. 36, no. 8, pp. 826-828, 2015. DOI : 10.1109/LED.2015.2442293.

[14] T. Sentaurus, "User guide, Version D-2010.03, March 2010-Synopsys," Inc., Mountain View, CA, 2010. DOI : https://www.synopsys.com/ silicon/tcad.

[15] P. V. Raja, J.-C. Nallatamby, N. DasGupta, and A. DasGupta, "Trapping effects on algan/gan hemt characteristics," Solid-State Electronics, vol. 176, p. 107929, 2021. DOI : 10.1016/j.sse.2020.107929.

[16] P. V. Raja, M. Bouslama, S. Sarkar, K. R. Pandurang, J.-C. Nallatamby, N. DasGupta, and A. Dasgupta, "Deep-level traps in AlGan/GaN and AlInn/GaN-based HEMTs with different buffer doping technologies," IEEE Transactions on Electron Devices, vol. 67, no. 6, pp. 2304-2310, 2020. DOI : 10.1109/TED.2020.2988439. 